\title{
Barriers to Cardiac Rehabilitation access of older heart failure patients and strategies for better implementation
}

\section{Analisi e strategie di riduzione delle barriere all'accesso del paziente scompensato ultra 75enne in Cardiologia Riabilitativa}

\author{
Giovanni Pulignano1, Maria Denitza Tinti', Donatella Del Sindaco², Stefano Tolone', \\ Giovanni Minardi', Antonio Lax², Massimo Uguccioni1 \\ 1 Heart Failure Clinic, Cardiology1/CCU, San Camillo Hospital, Rome \\ 2 Cardiology Unit, Nuovo Regina Margherita Hospital, Rome, Italy
}

\begin{abstract}
In heart failure (HF), cardiac rehabilitation (CR) may reduce decompensations, hospitalization, and ultimately mortality in long term. Many studies over the past decade have demonstrated that aerobic exercise training is effective and safe in stable patients with HF. Exercise CR resulted in a clinically important improvement in the QOL. Several clinical and psychosocial factors are associated with decreased participation in CR programs of elderly HF patients, such as perception of exercise as tiring or painful, comorbidities, lack of physician encouragement, and opinion that CR will not improve their health status. Besides low functional capacity, chronic deconditioning may also deter patients from participating in CR programs. Recent data suggest that current smoking, a BMI $\geq 30 \mathrm{~kg} / \mathrm{m}^{2}$, diabetes mellitus, and cognitive dysfunction are associated with failure to enroll in outpatient CR in older age group. Moreover the lack of availability of CR facilities or the absence of financial refunds for enrolment of CHF patients in cardiac rehabilitation programs can play a crucial role. Many of this factors are modifiable through patient education and self care strategy instruction, health providers sensibilization, and implementing economic measures in order to make CR affordable.
\end{abstract}

Corresponding author: Giovanni Pulignano, MD, Via G. Livraghi 1, 00152 Rome, Italy. Tel.: +39.06.58704562. E-mail: gipulig@yahoo.it

Key words: Heart failure; cardiac rehabilitation; elderly.

Parole chiave: Scompenso cardiaco; riabilitazione cardiovascolare; anziano.

Received for publication: 29 March 2016

Accepted for publication: 4 April 2016

(C) Copyright G. Pulignano et al., 2015

Tipografia PI-ME Editrice, Italy

Monaldi Archives for Chest Disease Cardiac Series 2015; 84:732

doi: 10.4081/monaldi.2015.732

This article is distributed under the terms of the Creative Commons Attribution Noncommercial License (by-nc 4.0) which permits any noncommercial use, distribution, and reproduction in any medium, provided the original author(s) and source are credited.

\section{Riassunto}

Numerosi studi hanno dimostrato come la riabilitazione cardiovascolare (RC) con esercizio aerobico sia risultato efficace e sicuro nei pazienti con scompenso cardiaco (SC), nel ridurre ospedalizzazioni, mortalità ed indurre un miglioramento della qualità di vita. Tuttavia numerosi fattori clinici e psicosociali, come la bassa capacità funzionale, le comorbidità, la percezione dell'esercizio fisico come noioso 0 doloroso, sono associati a ridotta partecipazione a RC da parte di pazienti anziani con SC. Inoltre dati recenti mostrano come l'abitudine tabagica, un BMI $\geq 30 \mathrm{~kg} / \mathrm{m}^{2}$, il diabete mellito ed il deterioramento cognitivo siano associati con il mancato arruolamento di pazienti anziani in programmi di RC. In aggiunta la mancanza di disponibilità di strutture per la RC o l'assenza di rimborsi finanziari per l'iscrizione dei pazienti con SC in programmi di riabilitazione cardiaca possono svolgere un ruolo cruciale. Molti di questi fattori risultano modificabili attraverso programmi di educazione sanitaria del paziente, sensibilizzazione del personale sanitario ed attraverso un'implementazione delle misure economiche al fine di rendere accessibile la RC.

\section{Introduction}

Heart failure (HF) is a significant and growing epidemic and its prevalence increases with age. In HF patients, cardiac rehabilitation (CR) improves dyspnea and fatigue, through effects on the cardiovascular and musculoskeletal systems. Moreover, CR may reduce hospitalizations, and ultimately mortality in the long term [1]. Many studies over the past decade have demonstrated that aerobic exercise training is effective and safe in stable patients with HF. Among such patients, randomized trials have demonstrated increases in peak oxygen consumption $\left(\mathrm{VO}_{2}\right)$ of $12 \%$ to $33 \%$ [2]. Benefits of aerobic exercise training in patients who have HF include improvement in autonomic and neurohormonal profile, peripheral blood flow, endotheliummediated flow-dependent vasodilation, skeletal muscle morphology and function.

Both the physiological aging process and HF are characterized by a reduced aerobic exercise capacity and by peak $\mathrm{VO}_{2}$. Peak $\mathrm{VO}_{2}$ declines per age decades in apparently healthy populations, with a steeper reduction after $70 \mathrm{~s}$ [3]. An impaired peak $\mathrm{VO}_{2}$ is characteristic of patients who have HF, mainly attributable to reduced muscle mass and peripheral vasoconstriction because of neurohormonal factors. As exercise capacity and muscle strength steady decline with age, elderly 
subjects are more exposed to the cardiovascular and peripheral changes associated with HF.

\section{Available evidence}

Apart from CR program characteristics, a recent Cochrane review including 33 trials of CR in HF patients shows that improvements in hospitalization and QOL with exercise-based CR are consistent regardless of the kind of CR program and may reduce mortality in the longer term. Compared with controls, exercise CR showed a trend towards a mortality reduction in trials with follow-up beyond 1 year (RR $0.88 ; 0.75$ to $1.02,0.09$ ). Exercise CR reduced the risk of overall (RR $0.75 ; 0.62$ to $0.92,0.005)$ and heart failure-specific hospitalisation (RR $0.61 ; 0.46$ to $0.80,0.0004)$ and resulted in a clinically important improvement in the Minnesota Living with Heart Failure questionnaire (mean difference: 5.8 points, -9.2 to $-2.4,0.0007$ ) [4].

Although multiple clinical trials have demonstrated favorable effects of aerobic exercise training in HF, most of them have enrolled predominantly younger male patients, excluding individuals with $\mathrm{HF}$ with preserved ejection fraction (HFpEF), women and elderly.

In the few exercise training trials that have included meaningful numbers of older patients who have HF, results have been generally favorable [5-7]. The largest exercise training trial involving older HF patients randomized 20060 - to 89- year old outpatients (mean 72 years, $34 \%$ women) in NYHA class II to III with a LVEF less than $40 \%$ to 24 weeks of exercise training or standard care. Significant improvement occurred in quality of life, NYHA class and 6-minute walk distance in exercisers, whereas no changes were reported in controls. In addition, fewer exercise patients (11\%) than standard care patients (20\%) were hospitalized during follow-up, although mortality was similarly low in both groups. Peak $\mathrm{VO}_{2}$ was not a measure outcome in this study. The low dropout rate (12\%) indicates that such a training program is feasible in most older patients who have HF [5].

$\mathrm{Pu}$ and colleagues randomized 16 women with $\mathrm{HF}$ of mean age 77 years to progressive resistance training for 10 weeks. At baseline, older HF women had approximately $40 \%$ lower muscle strength than those of similar age with other chronic diseases. Training was well tolerated and resulted in a $43 \%$ increase in strength and $13 \%$ increase in 6 minute walk distance but no increase in peak $\mathrm{VO}_{2}$. Older patients who have HF thus seem to derive significant increases in muscle strength and endurance from resistance training.

CR proved to be effective in improving peak and submaximal exercise capacity also in older patients with HFpEF, but in this particular subset of patients there is still a lack of consistent evidence of prognosis and QOL improvement [8].

Hence, the limited data available suggests that elderly subjects derive similar benefits from exercise training as younger subjects.

\section{Barriers to $\mathrm{CR}$ in the elderly}

In a recent survey by The Heart Failure Association, which involved more than 170 European cardiac centres, $40 \%$ centres following more than 36000 patients did not implement a CR program. This was mainly ascribed to the lack of resources (25\%), largely due to absence of staff or financial provision. The lack of a national or local referral protocol for such a program was the reason in $13 \%$ of the cases, and in $12 \%$ the perceived lack of evidence on safety or benefit was cited. When implemented, CR was proposed to all HF patients in only $55 \%$ of the centres, while referral was limited according to severity or aetiology [9]. Authors conclude that, despite increased awareness of the importance of a secondary prevention intervention in the $\mathrm{HF}$ population and increased availability of CR programs in HF in Europe, still too many patients are denied a highly recommended therapy, mainly due to scarce resources and logistics.

Among HF patients, older individuals are substantially less likely to be referred to CR, despite its proven benefits and the fact that guidelines recommend patients participate in CR program regardless of age [10], and that older patients adhere well to CR once enrolled [5].

In a US - Canadian survey addressing the CR barriers to enrollment and participation of outpatients with coronary artery disease, CR access was significantly related to age, with older patients ( $\geq 65$ years old) facing and reporting more barriers than younger patients, mostly related to health system organization and age issues, such as perception of exercise as tiring or painful, comorbidities, not knowing about CR, lack of physician encouragement, and opinion that CR will not improve their health status.

Conversely, younger patients more strongly endorsed work responsibilities and time constraints as CR barriers than older patients.

It is not surprising that elderly patients are less prone to accept a CR program, as they usually have lower exercise capacity, more comorbidities that may further impair functional capacity such as sarcopenia, COPD, osteoarthrosis, sensory impairments and cognitive dysfunction, and are less used to cope with chronic conditions. Also, they have less expectations in CR; facing the last aspect, physician encouragement and active motivation should play a key role in improving older patients' CR program participation. Moreover HF, as a chronic condition, requires optimal self care including active self-monitoring of symptoms and adherence to prescribed treatment, medications and daily routines recommendations; such behaviours are difficult to achieve in a population whose burden of frailty and cognitive impairment is high, and CR could represent the right setting for promoting self-care and behavioral changes [11]. Despite patient refusal and subjective perceived barriers, poor CR use rates among older adults is a result of the cumulative effect of many factors, including lack of physician referral, patient-related factors, or societal and economic difficulties.Physician referral is a key determinant of CR enrollment; failure to refer is probably the major cause of under-representation of older adults, especially those $\geq 75$ years of age in secondary CHD prevention programs. Barriers for physicians to recommend CR and enroll patients are related to individual beliefs and opinions, but also on the practitioners team and organization. Poor awareness of health providers about the efficacy of exercise might be one reason; thus, promoting information about the evidence and improving guideline adherence are a goal to reach in practitioners education.

By integrating CR into existing HF management programs and making referral part of quality assessment of HF management, CR might be prescribed more often and easier; this implementation, more extensively, should involve a multidisciplinary group of practitioners and dedicated figures (e.g., expert nurses, cardiologists, internists).

The lack of availability of CR facilities or the absence of financial refunds for enrolment of HF patients in cardiac rehabilitation programs can play a crucial role. To address such matters, dedicated economic and political measures are needed.

Low functional capacity, multiple comorbidities, and chronic deconditioning may also deter patients from participating in CR programs [12]. Recent data suggest that current smoking, a BMI $\geq 30 \mathrm{~kg} / \mathrm{m}^{2}$, diabetes mellitus, and cognitive dysfunction are associated with failure to enroll in outpatient CR in older age group [13]. In addition, patients may often have logistical or personal barriers to attend CR, including lack of transportation utilities or of a caregiver; financial commitment of CR program entry is another potential deterrent to CR participation by older adults. 
In an update of the previous Performance Measures on Cardiac Rehabilitation, in order to stress the importance of reducing the gap in delivering a high efficacy treatment such as CR to more persons with CVD who could benefit of, the AACVPR/ACC/AHA Cardiac Rehabilitation/Secondary Prevention Performance Measures Writing Committee states that even though some persons may have significant patient- or provider-oriented barriers to CR referral, nearly all patients with CVD can benefit from at least some components of a comprehensive, secondary prevention CR program, and that patient refusal should not be considered a reason not to provide a referral. For the same reason, medical factors different from life-threatening conditions should not considered as a contraindication for CR, as there are no a priori reasons to presume that a patient might not be able to participate in a rehabilitation and secondary prevention program [14]. According to the same evidences and intentions, the Heart Failure Association and the European Association for Cardiovascular Prevention and Rehabilitation recommends CR for HF secondary prevention also in older patients $[1,15]$. Also, the Joint ANMCO/IACPR-GICR Committee defines priority criteria based on clinical risk for admission to cardiac rehabilitation centers as inpatients, recommending CR as inpatients for mediumhigh risk patients.

In conclusion, cardiac rehabilitation programs are associated with significant health benefits, including improved survival, reduced hospitalizations and improved QOL. Several clinical and psychosocial factors are associated with decreased participation in cardiac rehabilitation programs of elderly HF patients. As many of this factors are modifiable through patient education and self care strategy instruction, health providers sensibilization, and implementing economic measures in order to make CR affordable, addressing these issues may improve participation and outcomes. Improved efforts are needed to refer and encourage eligible patients to attend and complete these programs; nevertheless, exercise prescription for older HF subjects should be individualized and tailored to a multidisciplinary comprehensive disease management program.

Still more studies are needed to investigate whether exercise training would have beneficial effect in elderly HF subjects with comorbidities, cognitive impairment and frailty.

Table 1. Key points.

- Cardiac rehabilitation (CR) is shown to improve patient outcomes, but many physicians do not always refer their elderly HF patients when indicated.

- Physician, patient and health system factors have roles in CR referral.

- The most relevant perceptions of physicians relate to CR programs' quality and benefit, and for patients relate to CR barriers.

- Patients might try to convey concerns during pre-referral CR, and physicians should be trained to identify and address them.

- Efforts to improve physicians' perceptions of CR and finding solutions to overcome CR barriers with patients could improve CR referral practice.

- More studies needed to investigate whether exercise training would have beneficial effect in elderly subjects.

\section{References}

1. Piepoli MF, Conraads V, Corrà U, et al. Exercise training in heart failure: From theory to practice. A consensus document of the Heart Failure Association and the European Association for Cardiovascular Prevention and Rehabilitation. Eur J Heart Fail 2011;13:347-57.

2. Piepoli MF, Flather M, Coats AJ. Overview of studies of exercise training in chronic heart failure: the need for a prospective randomized multicentre European trial. Eur Heart J 1998;19:830-41.

3. Fleg JL, Morrell CH, Bos AG, et al. Accelerated longitudinal decline of aerobic capacity in healthy older adults. Circulation 2005;112:674-82.

4. Sagar VA, Davies EJ, Briscoe S, et al. Exercise-based rehabilitation for heart failure: systematic review and meta-analysis. Open Heart 2015;2:e000163-e000163.

5. Austin J, Williams R, Ross L, et al. Randomised controlled trial of cardiac rehabilitation in elderly patients with heart failure. Eur $\mathrm{J}$ Heart Fail 2005;7(3 Spec. Iss.):411-7.

6. McKelvie RS, Teo KK, Roberts R, et al. Effects of exercise training in patients with heart failure: The Exercise Rehabilitation Trial (EXERT). Am Heart J 2002;144:23-30.

7. Tyni-Lenne R, Dencker K, Gordon A, et al. Comprehensive local muscle training increases aerobic working capacity and quality of life and decreases neurohormonal activation in patients with chronic heart failure. Eur J Heart Fail 2001;3:47-52.

8. Kitzman DW, Brubaker PH, Morgan TM, et al. Exercise training in older patients with Heart failure and preserved ejection fraction: A randomized, controlled, single-blind trial. Circ Hear Fail 2010;3:659-67.

9. Piepoli MF, Binno S, Corrà U, et al. ExtraHF survey: the first European survey on implementation of exercise training in heart failure patients. Eur J Heart Fail 2015;17:631-8.

10. Members AF, Mcmurray JJ V, Adamopoulos S, et al. ESC Guidelines for the diagnosis and treatment of acute and chronic heart failure 2012: The Task Force for the Diagnosis and Treatment of Acute and Chronic Heart Failure 2012 of the European Society of Cardiology. Developed in collaboration with the Heart. Eur Heart J 2012;33:1787-847.

11. Ades PA, Keteyian SJ, Balady GJ, et al. Cardiac rehabilitation exercise and self-care for chronic heart failure. JACC Hear Fail 2013;1:540-7.

12. Listerman J, Bittner V, Sanderson BK, Brown TM. Cardiac rehabilitation outcomes: impact of comorbidities and age. J Cardiopulm Rehabil Prev 2011;31:342-8.

13. Weingarten MN, Salz KA, Thomas RJ, Squires RW. Rates of enrollment for men and women referred to outpatient cardiac rehabilitation. J Cardiopulm Rehabil Prev 2011;31:217-22.

14. Thomas RJ, King M, Lui K, et al. AACVPR/ACCF/AHA 2010 update: performance measures on cardiac rehabilitation for referral to cardiac rehabilitation/secondary prevention services: a report of the American Association of Cardiovascular and Pulmonary Rehabilitation and the American College. Circulation 2010;122):1342-50.

15. Piepoli MF, Corrà U, Benzer W, et al. Secondary prevention through cardiac rehabilitation: from knowledge to implementation. A position paper from the Cardiac Rehabilitation Section of the European Association of Cardiovascular Prevention and Rehabilitation. Eur J Cardiovasc Prev Rehabil 2010;17:1-17. 